\title{
A Case of Early Keratoconus Associated with Eye Rubbing in a Young Child
}

Victoria Dimacali - Miltos Balidis - Aspasia Adamopoulou •

Athina Kozei · Nikolaos Kozeis

Received: December 3, 2019 / Published online: June 15, 2020

(C) The Author(s) 2020

\section{ABSTRACT}

Introduction: Keratoconus usually presents during puberty and is considered rare in young children.

Methods: Case report with clinical findings and computerized corneal tomography.

Results: We report the case of an 8-year-old girl with early bilateral keratoconus who presented with allergic conjunctivitis and persistent eye rubbing. Although our patient did not exhibit steep keratometry, early cones and inferotemporal thinnest corneal thicknesses were detected in both eyes using Scheimpflug imaging (Oculus GmbH Pentacam, Wetzlar, Germany). Belin/Ambrósio total $\mathrm{D}$ values were 1.85 on the right and 2.11 on the left. Improvement in best-

Digital Features To view digital features for this article go to https://doi.org/10.6084/m9.figshare.12340988.

V. Dimacali · M. Balidis - A. Adamopoulou ·

A. Kozei · N. Kozeis $(\square)$

Ophthalmica Institute of Ophthalmology and

Microsurgery, Thessaloniki, Greece

e-mail: kozeis@paidoofthamiatros.gr

V. Dimacali

Makati Medical Center, Metro Manila, Philippines

A. Kozei · N. Kozeis

Pediatric Eye Center of Greece, Thessaloniki, Greece

A. Kozei

School of Pharmacology, University of Nicosia,

Nicosia, Cyprus corrected visual acuity was noted after treatment of allergic eye disease, and corneal tomographic findings remained stable 4 months after initial consult.

Conclusion: This is a case of early diagnosed keratoconus in a young patient. Diagnosis of this condition in young children is challenging, as these patients are less likely to report visual complaints, and clinical examination is usually unremarkable. Keratoconus screening should be considered in children with atopy and eye rubbing behavior regardless of age, even in those with no other associated pathology and with negative family history.

Keywords: Allergic conjunctivitis; Atopy; Corneal tomography; Eye rubbing; Keratoconus; Pediatric keratoconus 


\section{Key Summary Points}

There are limited data at present regarding early keratoconus in young children, as the disorder is challenging to diagnose in this population.

Our 8-year old patient presented with decreased vision, allergic eye disease, and eye rubbing behavior. Tomography revealed normal keratometry values, mild asymmetric astigmatism, and early pachymetric and corneal elevation changes. Treatment of the allergy resulted in improvement of visual acuity, with tomographic findings remaining stable at four months.

Young children should be screened for keratoconus when presenting with decreased vision, atopy, and eye rubbing behavior, even in the absence of clinical corneal findings. The importance of avoiding eye rubbing should also be emphasized.

\section{INTRODUCTION}

Keratoconus (KC) is an ocular disorder in which there is progressive thinning and ectasia of the cornea due to alterations in its collagen structure [1]. It is associated with blurring of vision, light sensitivity, myopia, and irregular astigmatism, and may occasionally result in corneal scarring and acute corneal edema. Both eyes are affected although one eye may precede the other [2]. At present, no primary pathophysiologic explanation for keratoconus has been elucidated, as it is probably caused by various environmental, biomechanical, genetic, and biochemical disorders [3].

Keratoconus often presents during the second decade of life. It can be isolated or associated with other ocular and systemic disorders including vernal keratoconjunctivitis, atopy, Down syndrome, intellectual disability, and
Leber congenital amaurosis [3-5]. However, it is considered a rare condition in young children $[4,6,7]$.

Although vigorous eye rubbing seems to be a significant risk factor for keratoconus, the exact mechanism involved is still unknown $[3,4,8,9]$. Here we present the case of a young child with early keratoconus (EKC) with frequent eye rubbing due to allergic eye disease. Data regarding EKC in very young children are still limited, and any new reports are important. The study was conducted according to the tenets of the Declaration of Helsinki and was approved by the Ophthalmica Eye Institute Ethics Committee. The parents of the patient signed an informed consent form for publication of the case and the patient's clinical details.

\section{CASE PRESENTATION}

An 8-year-old girl was referred to our clinic for blurred vision in both eyes, reportedly starting 2 weeks prior to consult. Although her past ocular, medical, and family histories were otherwise unremarkable, her parents mentioned that she often rubbed her eyes vigorously. Her cycloplegic refraction was $+0.25 /-0.50 / 175^{\circ}$ and $+0.50 /-0.75 / 180^{\circ}$ for the right and left eyes, respectively, and her best-corrected visual acuity (BCVA) was 20/30 in both eyes. The findings from the ocular (clinical and paraclinical) and orthoptic examinations proved unremarkable. Both corneas were clear and had no clinical signs of ectasia, while the conjunctivae were mildly erythematous with some papillae.

Computerized corneal tomography was performed using rotating Scheimpflug imaging (Oculus GmbH Pentacam, Wetzlar, Germany). The curvature maps showed asymmetric bowtie astigmatism, with a significantly skewed radial axis of almost $60^{\circ}$ on the right, and asymmetric vertical $\mathrm{D}$ pattern astigmatism on the left (Fig. 1). Kmax was only 43.5D on the right and 44.1D on the left, and anterior astigmatism was $0.5 \mathrm{D}$ and $0.8 \mathrm{D}$, respectively. Pachymetry at the apex was $501 \mu \mathrm{m}$ on the right and $504 \mu \mathrm{m}$ on the left, with thinnest corneal thicknesses (TCT) of $496 \mu \mathrm{m}$ and $492 \mu \mathrm{m}$ 


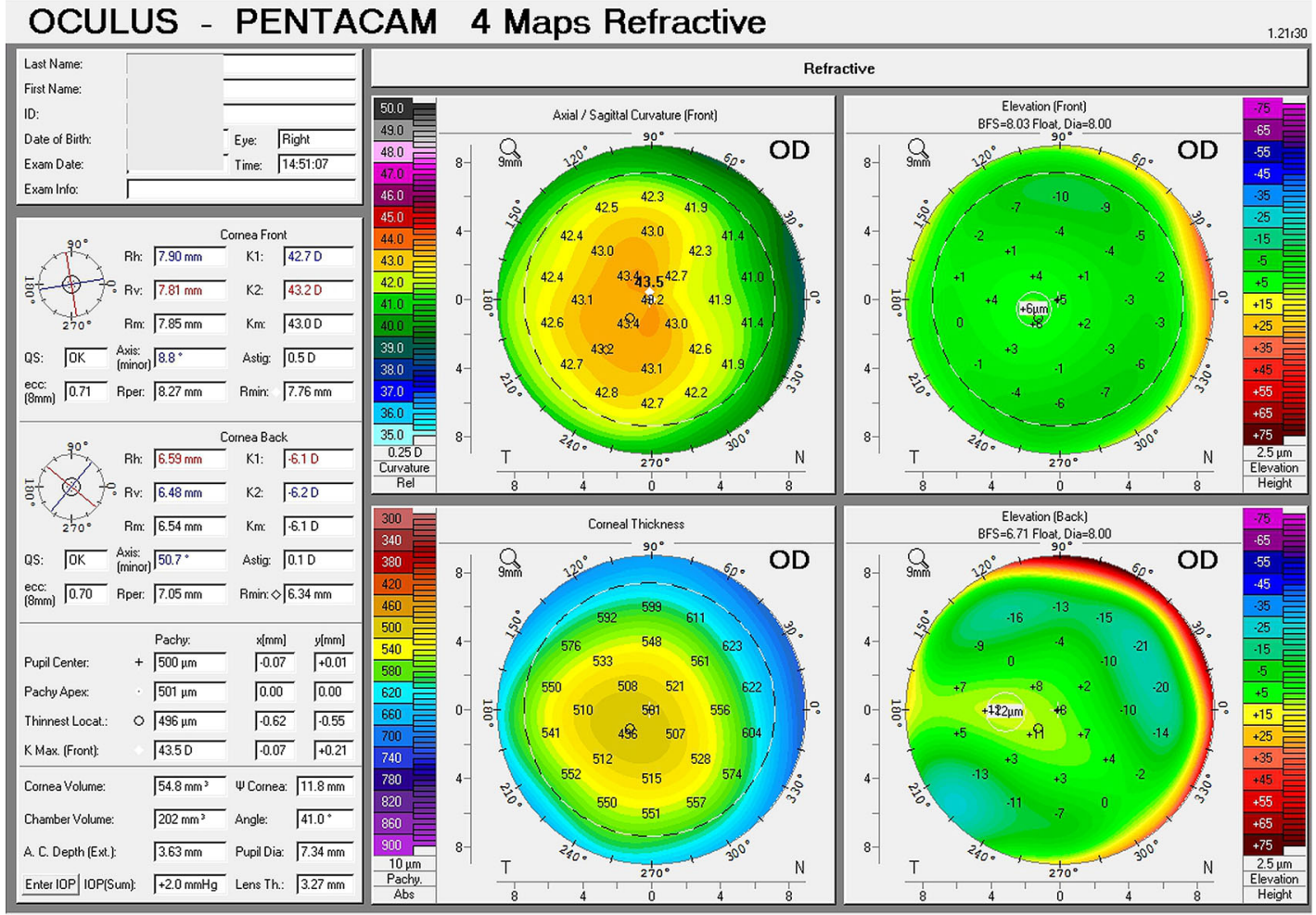

OCULUS - PENTACAM 4 Maps Refractive

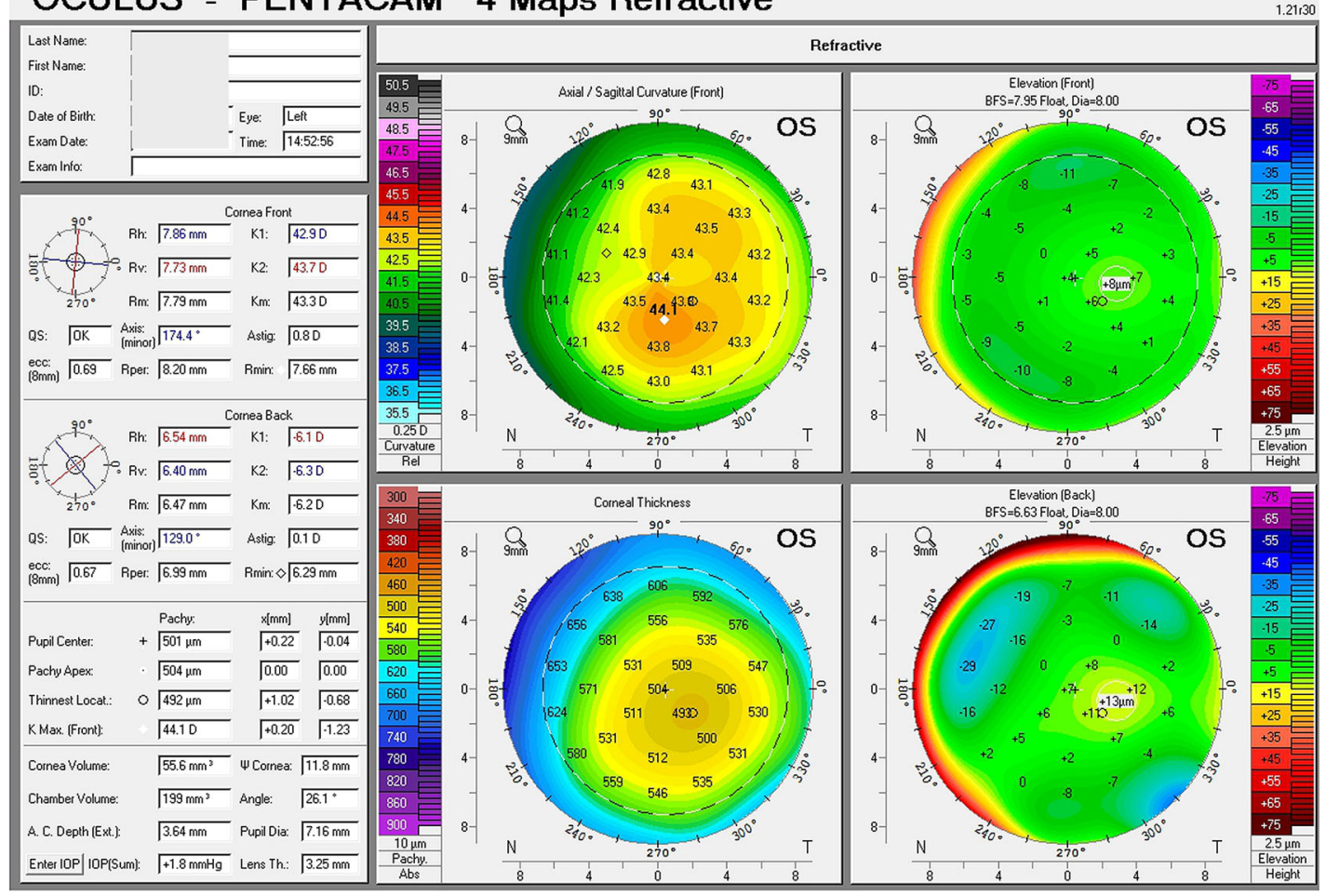

Fig. 1 Pentacam maps of both eyes taken at initial consult. Curvature maps show asymmetric bow-tie astigmatism on the right and asymmetric vertical $\mathrm{D}$ pattern astigmatism on the left 


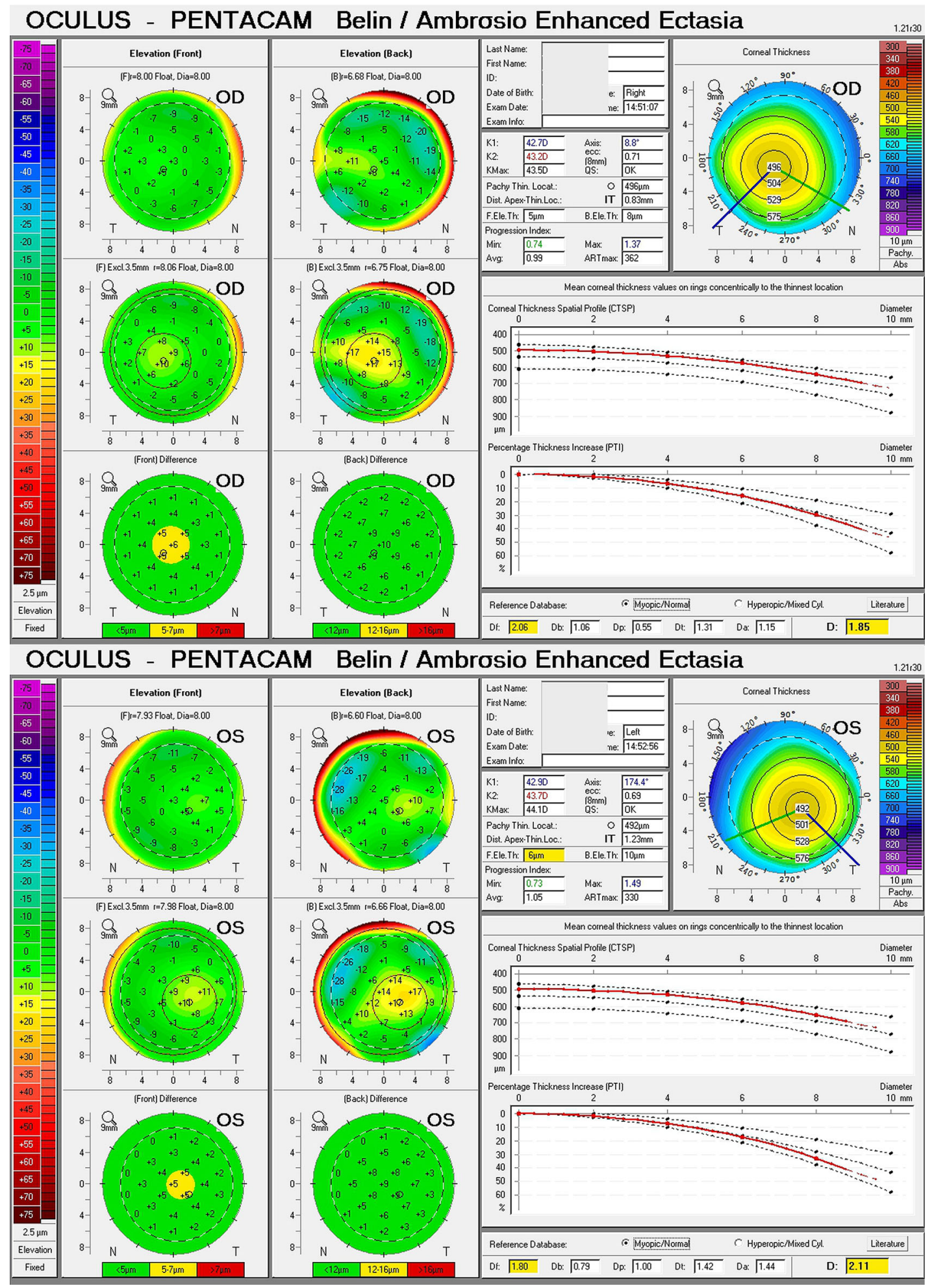

Fig. 2 Belin/Ambrósio Enhanced Ectasia display of both eyes at initial consult. The total D values were 1.85 on the right and 2.11 on the left. Pachymetric progression maps were within normal limits 
located inferotemporally in both eyes $[-0.62 /-0.55$ and $+1.02 /-0.68(x / y)$, respectively]. The Belin/Ambrósio Enhanced Ectasia Display total D values (BAD-D) were 1.85 on the right and 2.11 on the left (Fig. 2). Abnormalities were also detected in the anterior elevation maps of both eyes, as well as the anterior elevation value of the left eye at its thinnest point. Posterior elevation maps on best-fit sphere mode showed isolated islands in both eyes, with maximum elevation values corresponding to the TCT location in the left eye.

The BAD is a feature of the Pentacam, which uses data from elevation maps, pachymetry, best-fit sphere, and enhanced reference surface in a regression analysis to arrive at an overall " $\mathrm{D}$ " value, or BAD-D, which reflects the probability of ectasia [10]. The current version is the BAD III, which utilizes nine tomographic parameters [11]. Studies have shown that among the keratometric, pachymetric, and posterior elevation indices, the $\mathrm{D}$ value has the highest area under the receiver operating characteristic curve in differentiating between clinical and subclinical keratoconus (SKC) eyes and control eyes $[12,13]$.

A D value of 1.88 was found by one study to identify $99 \%$ of known KC cases, with a falsepositive rate of $2.5 \%$ [10]. Cut-off points in use for SKC in the literature are 1.45 by Ambrósio et al. [14], 1.54 by Hashemi et al. [15], and 1.61 by Ruiseñor Vazquez et al. [16]. A cross-sectional study which included SKC and KC eyes and normal eyes found that the best cut-off $\mathrm{D}$ value was 1.83 for identifying clinical $\mathrm{KC}$ from controls (100\% sensitivity and $96.0 \%$ specificity), and 1.73 for differentiating bilateral SKC eyes from normal eyes $(96.7 \%$ sensitivity and $79 \%$ specificity) [12].

The $\mathrm{D}$ values of our patient were greater than 1.73 in both eyes. The left eye, which had the higher BAD-D, also showed a suspicious vertical $\mathrm{D}$ pattern. This pattern has been proposed to reflect horizontal asymmetry in patients with suspected KC [17].

We treated the patient for 2 weeks with topical ketotifen $0.1 \mathrm{mg} / 0.4 \mathrm{~mL}$ twice a day, fluorometholone $0.1 \%$ four times a day, and preservative-free sodium hyaluronate gel four times a day, to reduce the discomfort and pruritus. She was also verbally encouraged by her parents to refrain from rubbing her eyes.

Two weeks later, the BCVA improved to $20 / 25$ on the right eye and $20 / 20$ on the left, and the refractive error decreased to $+0.25 \mathrm{D}$ sphere in both eyes. The parents reported that the child's eye rubbing had decreased significantly, so we decided to gradually taper off the medications and to do repeat imaging on the next follow-up.

Four months later, the BCVA was 20/20 in both eyes. The repeated imaging showed a slight increase in inferior steepening by $0.3 \mathrm{D}$ on the right and $0.2 \mathrm{D}$ on the left eye, while TCT and anterior and posterior elevation maps were stable (Fig. 3). We decided to have the patient follow up after 6 months and monitor her regularly thereafter.

\section{DISCUSSION}

Keratoconus usually presents during puberty and is relatively rare in young children. It is not typically suspected in the pediatric population when there are no other ocular and systemic associations and no family history. A large retrospective study of patients examined at a tertiary eye center in Lebanon found that only $0.53 \%$ of patients younger than 15 years of age were diagnosed with KC. The youngest patient with keratoconus was 9 years old. In general, those pediatric cases represented $2.96 \%$ of all keratoconus cases [6]. A retrospective study in a pediatric population in Saudi Arabia similarly detected keratoconus in $2.30 \%$ of patients 6 to 16 years of age [18]. The youngest patients with keratoconus reported so far in the literature are a 4-year-old girl from Turkey and a 4-year-old girl with Down syndrome from Switzerland $[4,7]$. Although limited data presently exists about EKC in young children, detection of ectatic disease at younger ages is expected to increase as imaging techniques become more advanced and awareness about screening improves. Our patient was 8 years old at presentation. Although she did not exhibit steep keratometry, Pentacam imaging of both eyes demonstrated localized steepening and an asymmetric bow-tie pattern, as well as 


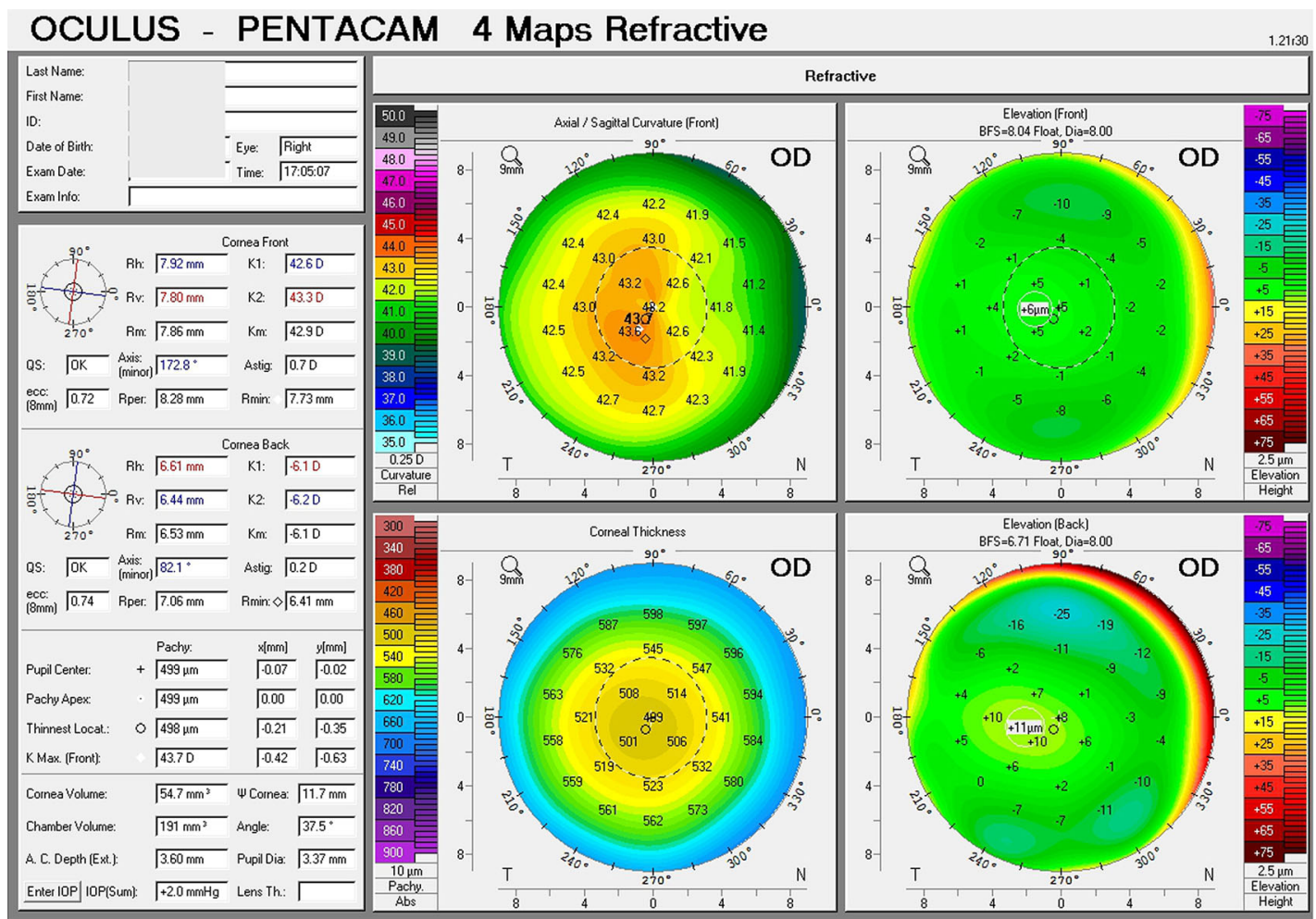

\section{OCULUS - PENTACAM 4 Maps Refractive}

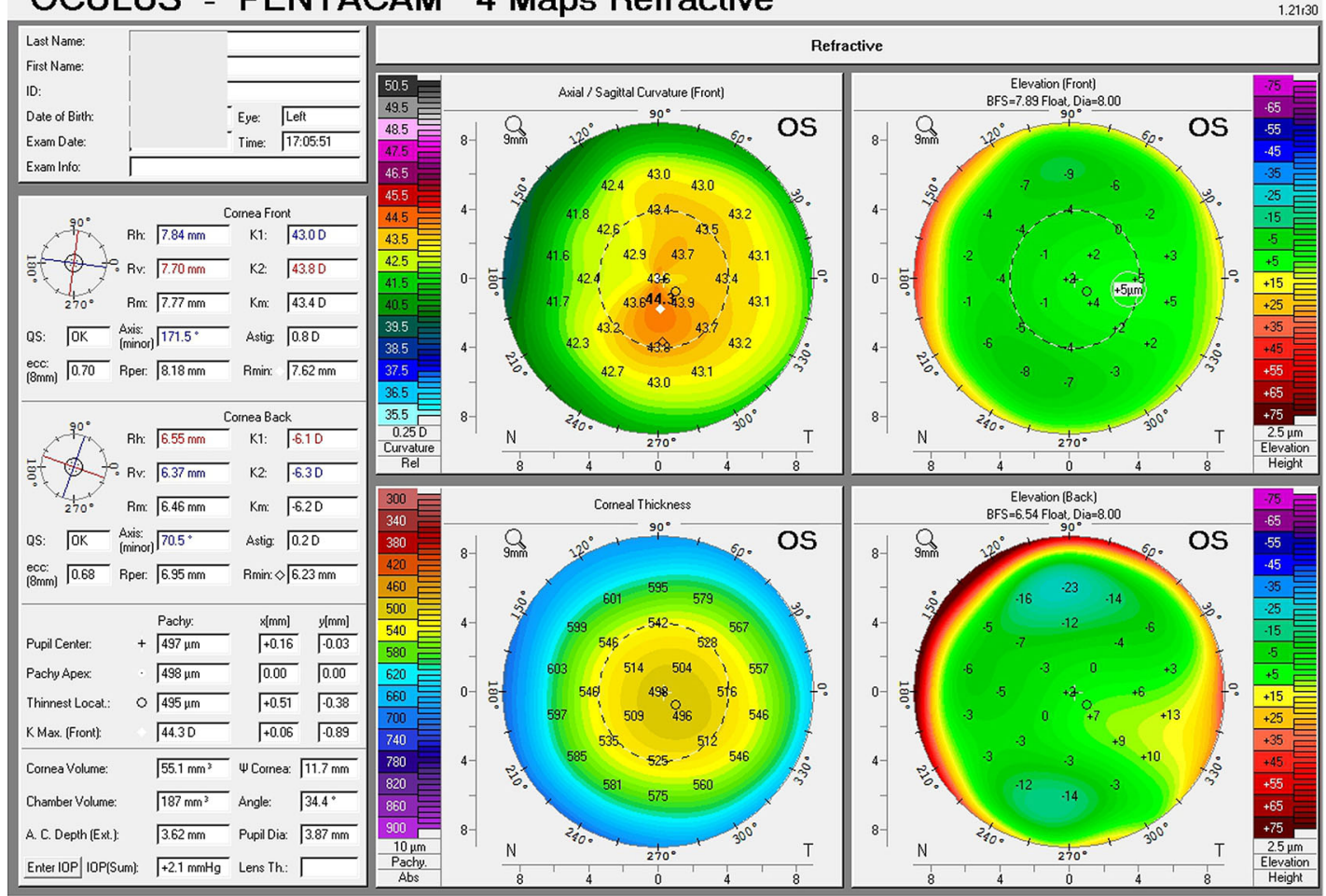

Fig. 3 Pentacam maps of both eyes taken 4 months after initial consult and initiation of medical therapy. Although there is mildly increased inferior steepening, TCT values and elevation maps remained stable in both eyes 
inferotemporally displaced thinnest corneal points. Central corneal thicknesses and TCTs were also less than $500 \mu \mathrm{m}$ in both eyes. This meets the standard diagnostic criteria proposed by Martinez-Abad et al. for SKC [19]. The main signs which must both be met are corneal topography with abnormal localized steepening or an asymmetric bow-tie pattern, and normalappearing cornea on slit lamp biomicroscopy. Complementary signs, one of which must be met, are the following: keratometric power greater than $47.0 \mathrm{D}$, oblique cylinder greater than 1.50D, central corneal thickness less than $500 \mu \mathrm{m}$, and clinical keratoconus in the fellow eye. Further investigation with corneal biomechanical analysis and epithelial mapping may also be done especially when clinical and tomographic findings are equivocal.

The 4-year-old girl from Turkey who was eventually diagnosed with keratoconus was a vigorous eye rubber presenting with decreased vision [4]. Our patient was similarly an eye rubber who presented with slightly reduced visual acuity. The association between eye rubbing and keratoconus is well known. A retrospective study found eye rubbing behavior to be present in $91.84 \%$ of patients diagnosed before age 15 , which was significantly higher than $70.06 \%$ of those diagnosed after age 27 [20]. Another study found eye rubbing to be present in $79 \%$ of a pediatric population, with all patients showing clinical signs of allergic eye disease [5]. Although the exact mechanism by which keratoconus may develop remains to be elucidated, McMonnies proposed the following responses to occur due to eye rubbing: increase in corneal temperature, epithelial thinning, increased inflammatory mediators in the tear film, abnormal enzyme activity, intraocular pressure spikes, high hydrostatic tissue pressure, thixotropic decrease in ground substance viscosity, temporary displacement of ground substance from the corneal apex, curvature transfer to the cone apex, slippage between collagen fibrils at the cone apex, changes to keratocytes, and corneal scarring [21]. Eye rubbing has also been shown to raise the levels of tear metalloproteinase-13, IL- 6 , and TNF- $\alpha$ in both normal and keratoconic eyes [1].
Ectasia progression has been defined as steepening of the anterior or posterior corneal surface, or a thinning and/or an increase in the rate of corneal thickness change from the periphery to the thinnest point, with the changes being consistent over time and above the normal variability of the measurement system. A decrease in best-spectacle-corrected visual acuity, although often noted, is not necessary in documenting progression [3]. In our case, corneal tomographic findings remained stable over the next 4 months. In the case of the 4-year-old girl from Switzerland with Down syndrome who had rapid progression of keratoconus, stabilization of the tomographic measurements and visual acuity was achieved after consecutive bilateral collagen cross-linking [7].

Corneal collagen cross-linking (CXL) must be offered when any sign of progression is noted. The efficacy and safety of CXL in adults has been well established, but experience in the pediatric population is limited. Recent studies have demonstrated that conventional epithelium-off CXL is safe and effective in preventing progression for up to 10 years [22]. Accelerated CXL has also been shown by some studies to be safe and effective in children in slowing or preventing the progression of keratoconus for up to 3 years [23-25].

Early detection of keratoconus and its subclinical forms in young children is important albeit challenging, especially in patients with no evident risk factors or family history. Advanced cases at the time of diagnosis usually progress more rapidly in young children than in adults $[6,26,27]$. Nonsurgical management of $\mathrm{KC}$ should include verbal guidance to avoid eye rubbing, use of topical anti-allergic medication in patients with allergy, and use of lubricants for ocular irritation [3]. In early cases, control of the allergic reaction to decrease the eye rubbing impulse may reduce the inflammation and help avoid corneal complications [28]. Salomao et al. [29] stress the need for screening; though moderate and advanced cases of keratoconus are easily recognized, identification of mild and early forms of keratoconus remains a challenge. Ambrósio et al. [30] note that control of allergy and inflammation of the ocular surface is important, and new advances in diagnosis 
based on artificial intelligence, genetics, and other methods will increase the accuracy of diagnosis and lead to customized treatment planning. Our patient's visual acuity improved and her tomographic profile remained stable just with conservative treatment. With this case, we would like to highlight the entity of EKC in very young children and the importance of screening in specific patients, since young children are less likely to report functional complaints and the cornea, by definition, appears normal upon clinical examination. The combination of suspicious eye rubbing behavior and symptoms should prompt further investigation.

\section{ACKNOWLEDGEMENTS}

We would like to thank our patient and her parents for giving their consent for the publication of this case.

Funding. No funding or sponsorship was received for this study or publication of this article.

Authorship. All named authors meet the International Committee of Medical Journal Editors (ICMJE) criteria for authorship for this article, take responsibility for the integrity of the work as a whole, and have given their approval for this version to be published.

Disclosures. The authors Victoria Dimacali, Aspasia Adamopoulou, Miltos Balidis and Athina Kozei declare that they have no conflict of interest. Nikolaos Kozeis is a member of the journal's Editorial Board. It should also be noted that after the completion of the manuscript, the following occurred: author Nikolaos Kozeis changed his affiliation from "Ophthalmica" to "Pediatric Eye Centre of Greece, Thessaloniki, Greece". Athina Kozei changed one of her affiliations from "Ophthalmica" to "Pediatric Eye Center of Greece, Thessaloniki, Greece", and she retained the second one, School of Pharmacology, University of Nicosia; Nicosia, Cyprus.
Compliance with Ethics Guidelines. The study was conducted according to the tenets of the Declaration of Helsinki and was approved by the Ophthalmica Eye Institute Ethics Committee. The parents of the patient signed an informed consent form for publication of the case and the patient's clinical details.

Data Availability. Data sharing is not applicable for this article as no data sets were generated or analyzed during the current study.

Open Access. This article is licensed under a Creative Commons Attribution-NonCommercial 4.0 International License, which permits any non-commercial use, sharing, adaptation, distribution and reproduction in any medium or format, as long as you give appropriate credit to the original author(s) and the source, provide a link to the Creative Commons licence, and indicate if changes were made. The images or other third party material in this article are included in the article's Creative Commons licence, unless indicated otherwise in a credit line to the material. If material is not included in the article's Creative Commons licence and your intended use is not permitted by statutory regulation or exceeds the permitted use, you will need to obtain permission directly from the copyright holder. To view a copy of this licence, visit http://creativecommons.org/licenses/by$\mathrm{nc} / 4.0 /$.

\section{REFERENCES}

1. Mukhtar S, Ambati BK. Pediatric keratoconus: a review of the literature. Int Ophthalmol. 2018;38(5):2257-66.

2. Loukovitis E, Sfakianakis K, Syrmakesi P, et al. Genetic aspects of keratoconus: a literature review exploring potential genetic contributions and possible genetic relationships with comorbidities. Ophthalmol Ther. 2018;7(2):263-92.

3. Gomes JA, Tan D, Rapuano CJ, et al. Global consensus on keratoconus and ectatic diseases. Cornea. 2015;34(4):359-69.

4. Gunes A, Tok L, Tok Ö, Seyrek L. The youngest patient with bilateral keratoconus secondary to 
chronic persistent eye rubbing. Semin Ophthalmol. 2015;30(5-6):454-6.

5. Hamilton A, Wong S, Carley F, Chaudhry N, Biswas $\mathrm{S}$. Tomographic indices as possible risk factors for progression in pediatric keratoconus. J AAPOS. 2016;20(6):523-6.

6. El-Khoury S, Abdelmassih Y, Hamade A, et al. Pediatric keratoconus in a tertiary referral center: incidence, presentation, risk factors, and management. J Refract Surg. 2016;32(8):534-41.

7. Saabti S, Tappeiner C, Frueh BE. Corneal crosslinking in a 4-year-old child with keratoconus and Down syndrome. Cornea. 2015;34(9):1157-60.

8. Bawazeer AM, Hodge WG, Lorimer B. Atopy and keratoconus: a multivariate analysis. Br J Ophthalmol. 2000;84(8):834-6.

9. Panahi-Bazaz MR, Sharifipour F, Moghaddasi A. Bilateral keratoconus and corneal hydrops associated with eye rubbing in a 7-year-old girl. J Ophthalmic Vis Res. 2014;9(1):101-5.

10. Imbornoni LM, McGhee CNJ, Belin MW. Evolution of keratoconus: from diagnosis to therapeutics. Klin Monbl Augenheilkd. 2018;235(6):680-8.

11. Cavas-Martinez F, De la Cruz SE, Nieto Martinez J, Fernandez Cañavate FJ, Fernandez-Pacheco DG. Corneal topography in keratoconus: state of the art. Eye Vis (Lond). 2016;3:5.

12. Huseynli S, Abdulaliyeva F. Evaluation of Scheimpflug tomography parameters in subclinical keratoconus, clinical keratoconus, and normal Caucasian eyes. Turk J Ophthalmol. 2018;48(3): 99-108.

13. Muftuoglu O, Ayar O, Hurmeric V, Orucoglu F, Kilic I. Comparison of multimeric D index with keratometric, pachymetric and posterior elevation parameters in diagnosing subclinical keratoconus in fellow eyes of asymmetric keratoconus patients. J Cataract Refract Surg. 2015;41(3):557-65.

14. Ambrósio R Jr, Faria-Correia F, Ramos I, et al. Enhanced screening for ectasia susceptibility among refractive candidates: the role of corneal tomography and biomechanics. Curr Ophthalmol Rep. 2013;1(1):28-38.

15. Hashemi H, Beiranvand A, Yekta A, Maleki A, Yazdani N, Khabazkhoob M. Pentacam top indices for diagnosing subclinical and definite keratoconus. J Curr Ophthalmol. 2016;28(1):21-6.

16. Ruiseñor Vazquez PR, Galletti JD, Minguez N, et al. Pentacam Scheimpflug tomography findings in topographically normal patients and subclinical keratoconus cases. Am J Ophthalmol. 2014;158(1): $32-40$.

17. Abad JC, Rubinfeld RS, Del Valle M, Belin MW, Kurstin JM. A novel topographic pattern in some keratoconus suspects. Ophthalmology. 2007;114(5):1020-6.

18. Netto EA, Al-Otaibi WM, Hafezi NL, Kling S, AlFarhan HM, et al. Prevalence of keratoconus in paediatric patients in Riyadh, Saudi Arabia. Br J Ophthalmol. 2018;102:1436-41.

19. Martinez-Abad A, Piñero DP. New perspectives on the detection and progression of keratoconus. J Cataract Refract Surg. 2017;43(9):1213-27.

20. Léoni-Mesplié S, Mortemousque B, Mesplié N, Touboul D, Praud D, et al. Epidemiologial aspects of keratoconus in children. J Fr Ophthalmol. 2012;35(10):776-85.

21. McMonnies CW, Boneham GC. Keratoconus, allergy, itch, eye-rubbing and hand-dominance. Clin Exp Optom. 2003;86(6):376-84.

22. Mazzotta C, Traversi C, Baiocchi S, et al. Corneal collagen cross-linking with riboflavin and ultraviolet A light for pediatric keratoconus: ten-year results. Cornea. 2018;37(5):560-6.

23. Sarac O, Kosekahya P, Caglayan M, Tanriverdi B, Taslipinar Uzel AG, Cagil N. Mechanical versus transepithelial phototherapeutic keratectomy epithelial removal followed by accelerated corneal crosslinking for pediatric keratoconus: long-term results. J Cataract Refract Surg. 2018;44(7):827-35.

24. Ulusoy DM, Göktaş E, Duru N, et al. Accelerated corneal crosslinking for treatment of progressive keratoconus in pediatric patients. Eur J Ophthalmol. 2017;27(3):319-25.

25. Badawi AE. Accelerated corneal collagen crosslinking in pediatric keratoconus: one year study. Saudi J Ophthalmol. 2017;31(1):11-8.

26. Léoni-Mesplié S, Mortemousque B, Touboul D, Malet F, Praud D, et al. Scalability and severity of keratoconus in children. Am J Ophthalmol. 2012;154(1):56-62.

27. Perez-Straziota C, Gaster RN, Rabinowitz YS. Corneal cross-linking for pediatric keratoconus review. Cornea. 2018;37(6):802-9.

28. Shetty R, Sureka S, Kusumgar P, Sethu S, Sainani K. Allergen-specific exposure associated with high immunoglobulin $\mathrm{E}$ and eye rubbing predisposes to progression of keratoconus. Indian J Ophthalmol. 2017;65(5):399-402. 
29. Dawson G, Ambrósio R. Recent developments in keratoconus diagnosis. Expert Rev Ophthalmol. 2018;13(6):329-41.
30. Ambrósio R Jr, Lopes B, Amara J, Correia FF, Canedo ALC, Salomão M, da Silva RS, Sena N Jr. Keratoconus: Breaking paradigms and contradictions of a new subspecialty. Rev Bras Oftalmol. 2019;78(2): 81-5. 